\title{
Automatic Detection and Quantification of Acute Cerebral Infarct by Fuzzy Clustering and Histographic Characterization on Diffusion Weighted MR Imaging and Apparent Diffusion Coefficient Map
}

\author{
Jang-Zern Tsai, ${ }^{1}$ Syu-Jyun Peng, ${ }^{1}$ Yu-Wei Chen, ${ }^{2,3,4}$ Kuo-Wei Wang, ${ }^{1,5}$ \\ Hsiao-Kuang Wu, ${ }^{2}$ Yun-Yu Lin, ${ }^{3}$ Ying-Ying Lee, ${ }^{3}$ Chi-Jen Chen, ${ }^{6}$ Huey-Juan Lin, 7 \\ Eric Edward Smith, ${ }^{8}$ Poh-Shiow Yeh, ${ }^{7}$ and Yue-Loong Hsin ${ }^{9,10,11}$ \\ ${ }^{1}$ Department of Electrical Engineering, National Central University, Jhongli City, Taoyuan County 32001, Taiwan \\ ${ }^{2}$ Department of Computer Science and Information Engineering, National Central University, Jhongli City, \\ Taoyuan County 32001, Taiwan \\ ${ }^{3}$ Department of Neurology, Landseed Hospital, Pingzhen City, Taoyuan County 32449, Taiwan \\ ${ }^{4}$ Department of Neurology, National Taiwan University Hospital, Taipei City 10002, Taiwan \\ ${ }^{5}$ Department of Medical Imaging, Landseed Hospital, Pingzhen City, Taoyuan County 32449, Taiwan \\ ${ }^{6}$ Department of Radiology, Taipei Medical University-Shuang Ho Hospital, New Taipei City 23561, Taiwan \\ ${ }^{7}$ Department of Neurology, Chi-Mei Medical Center, Tainan City 71004, Taiwan \\ ${ }^{8}$ Department of Clinical Neurosciences and Hotchkiss Brain Institute, University of Calgary, Calgary, AB, Canada T2N 1N4 \\ ${ }^{9}$ Epilepsy Center, Buddhist Tzu Chi General Hospital, Hualian City, Hualian County 97002, Taiwan \\ ${ }^{10}$ Biomedical Electronics Translational Research Center, National Chiao Tung University, Hsinchu City 30010, Taiwan \\ ${ }^{11}$ Department of Neurology, Chung Shan Medical University Hospital, Taichung City 40201, Taiwan
}

Correspondence should be addressed to Yu-Wei Chen; yuwchen@gmail.com

Received 5 November 2013; Revised 31 December 2013; Accepted 9 January 2014; Published 12 March 2014

Academic Editor: George Pengas

Copyright (C) 2014 Jang-Zern Tsai et al. This is an open access article distributed under the Creative Commons Attribution License, which permits unrestricted use, distribution, and reproduction in any medium, provided the original work is properly cited.

\begin{abstract}
Determination of the volumes of acute cerebral infarct in the magnetic resonance imaging harbors prognostic values. However, semiautomatic method of segmentation is time-consuming and with high interrater variability. Using diffusion weighted imaging and apparent diffusion coefficient map from patients with acute infarction in 10 days, we aimed to develop a fully automatic algorithm to measure infarct volume. It includes an unsupervised classification with fuzzy C-means clustering determination of the histographic distribution, defining self-adjusted intensity thresholds. The proposed method attained high agreement with the semiautomatic method, with similarity index $89.9 \pm 6.5 \%$, in detecting cerebral infarct lesions from 22 acute stroke patients. We demonstrated the accuracy of the proposed computer-assisted prompt segmentation method, which appeared promising to replace the laborious, time-consuming, and operator-dependent semiautomatic segmentation.
\end{abstract}

\section{Introduction}

Cerebrovascular disease is one of the leading causes of acute mortality and chronic disability [1]. The volume of infarct is associated with severity of acute ischemic stroke and correlates with clinical prognosis and the effect of endovascular therapy [2-4]. A rapid and reliable method of determination of volume of acute infarct will help predict the prognosis and facilitate further investigation.

The diffusion weighted imaging (DWI) is more sensitive than other magnetic resonance imaging (MRI) modalities to small water diffusion changes in the acute ischemic brain, especially within 48 hours of the ictus [5-9]. 


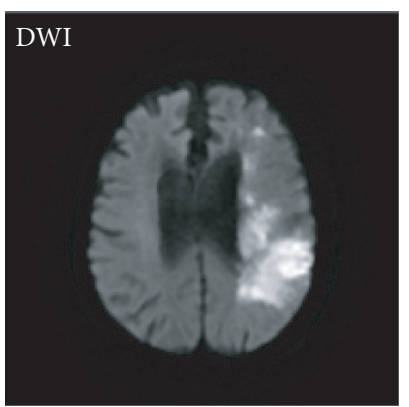

(a)

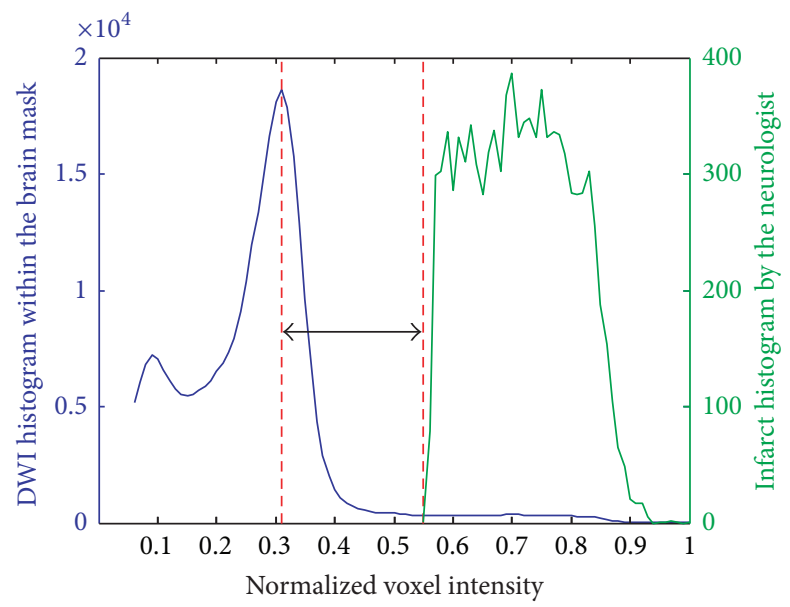

(e)

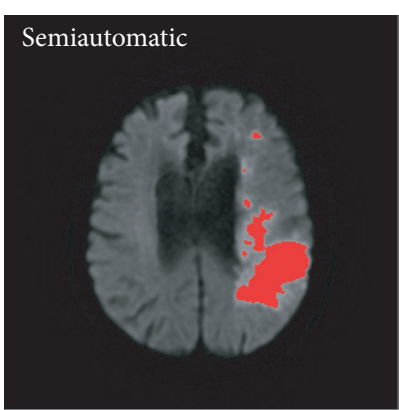

(b)

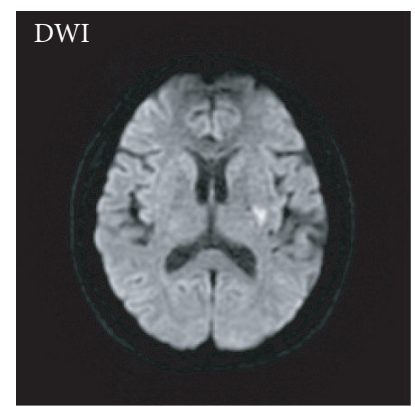

(c)

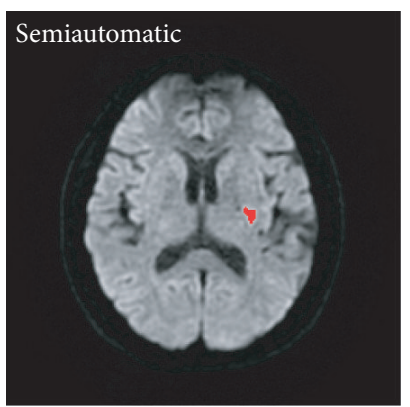

(d)

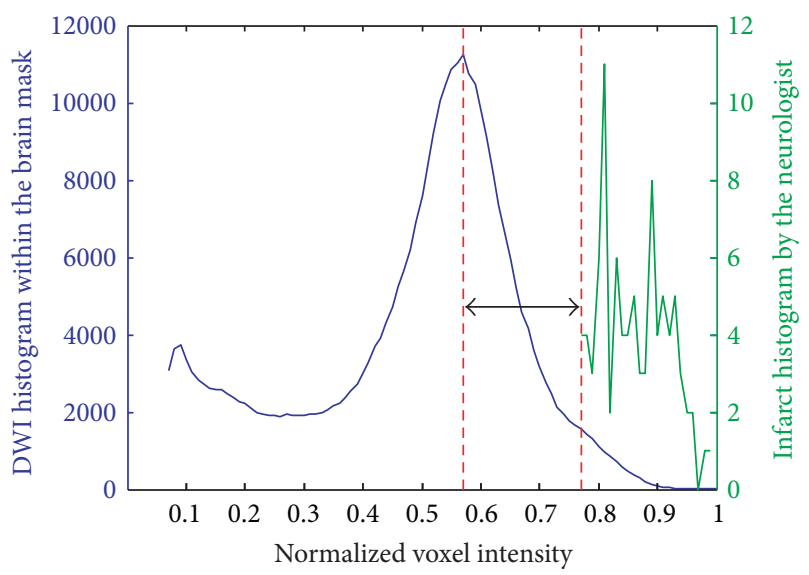

(f)

FIgURE 1: The histographic characteristics of the raw DWI and the infarct. (a) The raw DWI of patient number 20. (b) The semiautomatically demarcated infarcts, printed in red, of patient number 20 . The infarct volume $=46.828 \mathrm{~mL}$. The lower bound of the infarcts $=I_{\text {peak }}+0.24$. $(\mathrm{c})$ The raw DWI of patient number 3. (d) The semiautomatically demarcated infarcts, printed in red, of patient number 3 . The infarct volume $=0.399 \mathrm{~mL}$. The lower bound of the infarcts $=I_{\text {peak }}+0.21$. (e) The histogram of the normalized voxel intensity within the brain mask of the whole-brain DWI and the intensity distribution of the semiautomatically demarcated infarcts of patient number 20. (f) The histogram of the normalized voxel intensity within the brain mask of the whole-brain DWI and the intensity distribution of the semiautomatically demarcated infarcts of patient number 3 .

Automatic algorithms for segmentation for acute infarct in MRI have been reported [10-15]. The unsupervised method developed by Li et al. was based on a multistage procedure including image preprocessing, calculation of tensor field, measurement of diffusion anisotropy, segmentation of infarct volume based on adaptive multiscale statistical classification, and partial volume voxel reclassification [11]. Bhanu Prakash et al. used a probabilistic neural network for selecting infarct slices and an adaptive Gaussian mixture model for segmentation of the infarcts [12]. Hevia-Montiel et al. developed a method for cerebral infarct lesion segmentation from DWI by applying nonparametric density estimation [13]. Gupta et al. identified the infarct slices and the hemisphere automatically in DWI based on the difference in the percentile characteristics of intensity normalized images and parameters of infarct slice identification and infarct hemisphere identification [14]. Shen et al. detected infarct lesions based on the voxel intensity segmentation and the spatial location of tissue distribution [15].

We aimed to design a DWI-based computer-assisted method to provide clinicians a prompt and accurate determination of the volumes of acute cerebral infarct. The operation of this method is based on the histographic characteristic of the output clusters of a fuzzy C-means (FCM) clustering [16]. Additional measures were taken to ensure the accuracy of infarct detection, including discriminating infarcts from artifacts due to magnetic inhomogeneity by incorporating the histographic information in the apparent diffusion coefficient (ADC) map.

\section{Materials and Methods}

2.1. Subjects and Image Acquisition. Landseed Hospital has been participating in the nationwide Taiwan Stroke Registry, which prospectively registered patients with stroke onset within 10 days according to a preestablished system [17]. For this study, we recruited 22 patients (11 women and 11 men, 62-83 years of age) with acute cerebral infarction and MRI examinations during January-February 2011. The protocol of this research has been reviewed and approved by the Institutional Review Board (IRB) of Landseed Hospital.

All MRIs were acquired with a Signa HDxt 1.5T Optima edition (GE Healthcare, Waukesha, WI) and consisted 


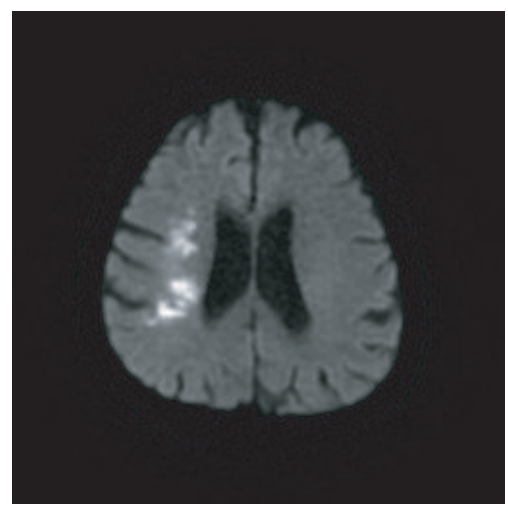

(a)

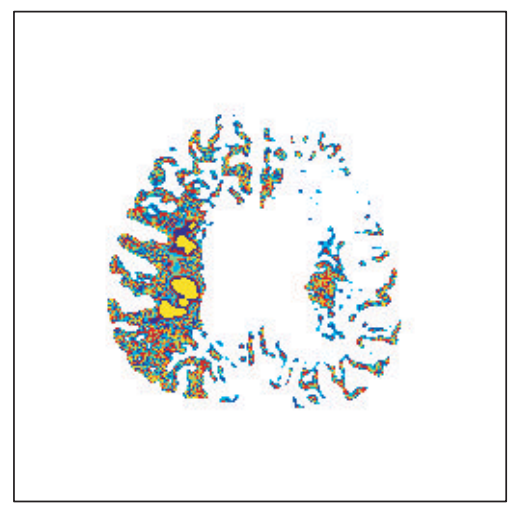

(d)

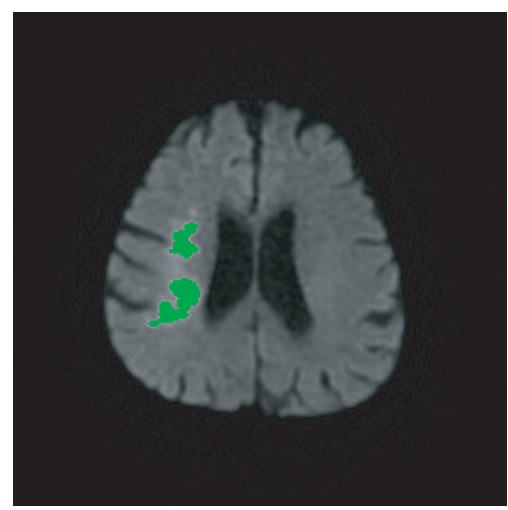

(g)

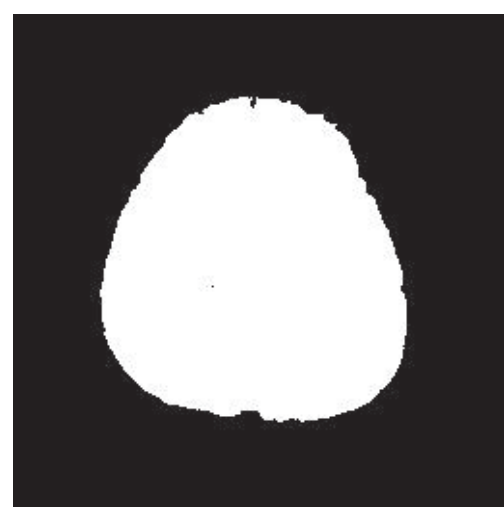

(b)

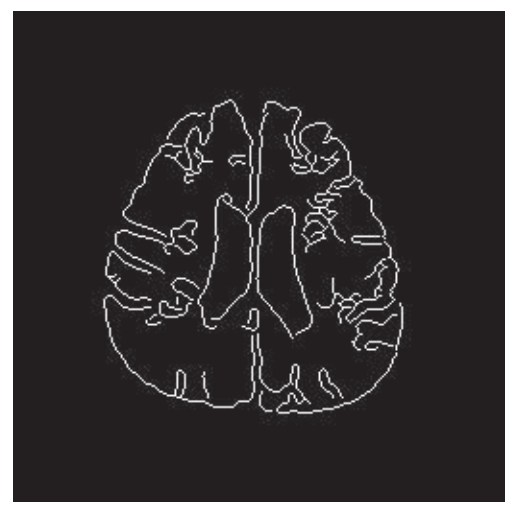

(e)

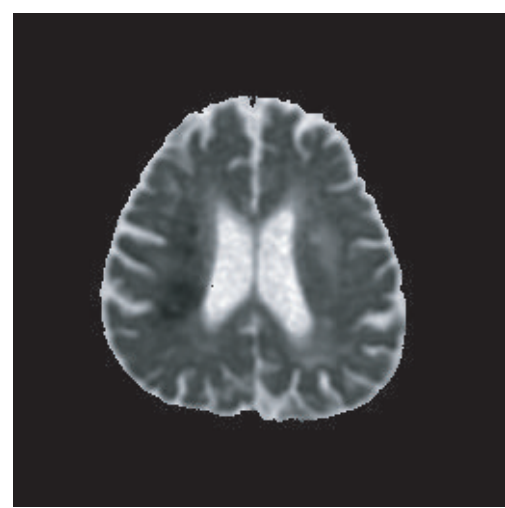

(h)

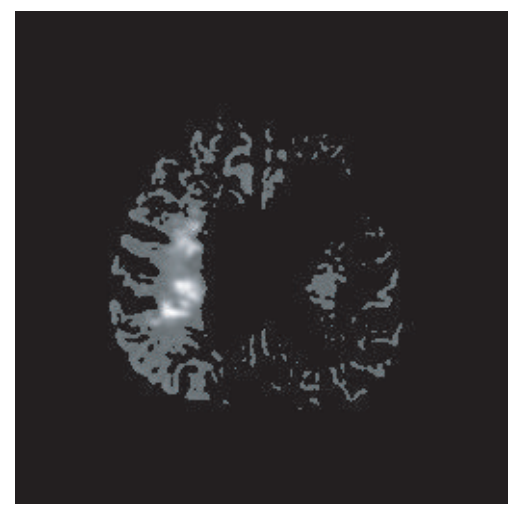

(c)

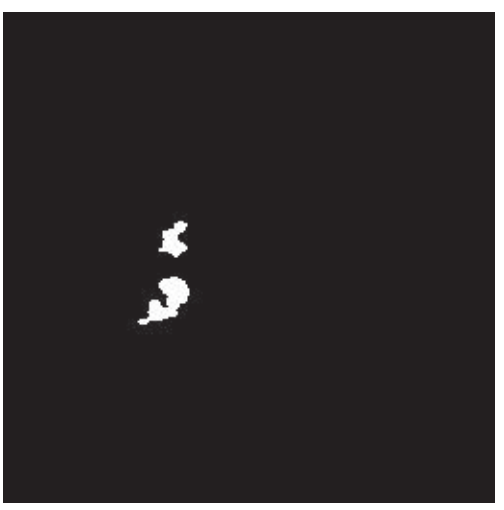

(f)

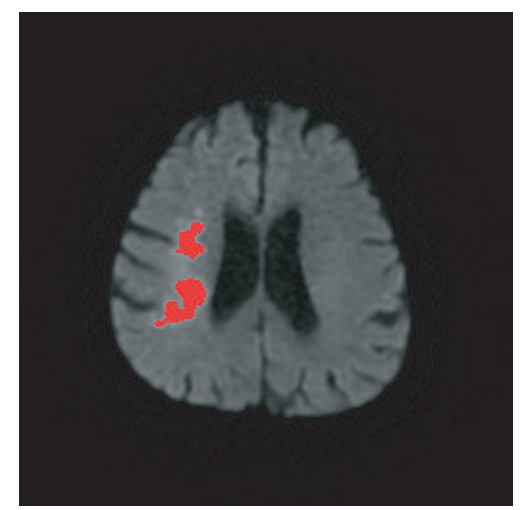

(i)

FIGURE 2: Illustrating the procedure of the proposed method with exemplary images. (a) The raw DWI. (b) The brain mask. (c) The regionof-interest map derived after the preclustering elimination in Step 3. (d) The FCM cluster map for the 50 clusters in Step 4 . (e) Canny edge detection map. (f) The computer-assisted infarct segmentation result. (g) The detected infarct was mapped to raw DWI. (h) The ADC map. (i) The semiautomatically demarcated infarct regions by the experienced neurologist.

of a DWI scan (TR/TE/Flip angle $=6000 \mathrm{~ms} / 82.8 \mathrm{~ms} / 90^{\circ}$, FOV $=230 \times 230 \mathrm{~mm}^{2}$, matrix $=128 \times 128$, in-plane resolution $=1.79 \times 1.79 \mathrm{~mm}, 24$ axial slices, $5 \mathrm{~mm}$ slice thickness with $1 \mathrm{~mm}$ gap) and an ADC map with $b=1000 \mathrm{~s} / \mathrm{mm}^{2}$.

2.2. Automatic Infarct Detection Procedure. The proposed method was developed on a personal computer with Intel Core i5 CPU, $2.67 \mathrm{GHz}$ processor speed, and $4 \mathrm{~GB}$ RAM. The infarct detection procedure was carried out mainly with a MATLAB program (The MathWorks, Inc., Natick, MA). We utilized the histographic characteristic of the DWI for infarct detection. Figure 1 illustrates one example with a large infarct volume and another with a small infarct volume. The procedure comprised the following steps.

Step 1 (coregistration and intensity normalization). The ADC map was registered to the corresponding DWI by a rigid registration (translation and rotation) and a trilinear interpolation based on the normalized mutual information method 


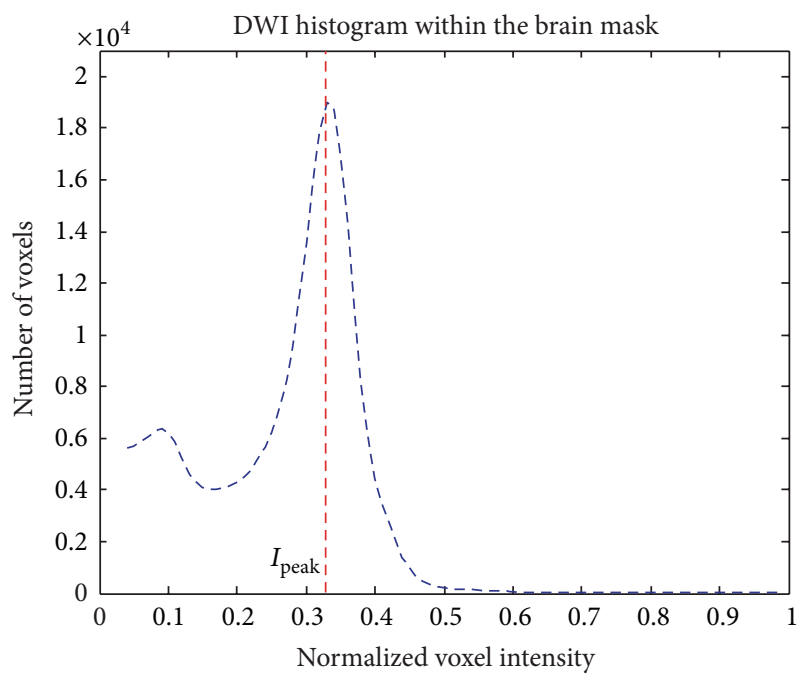

(a)

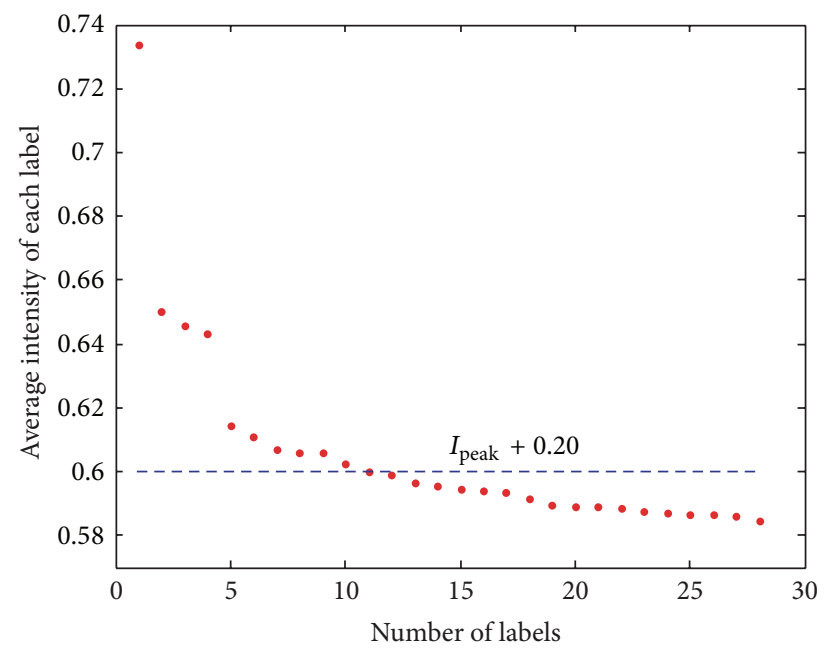

(c)

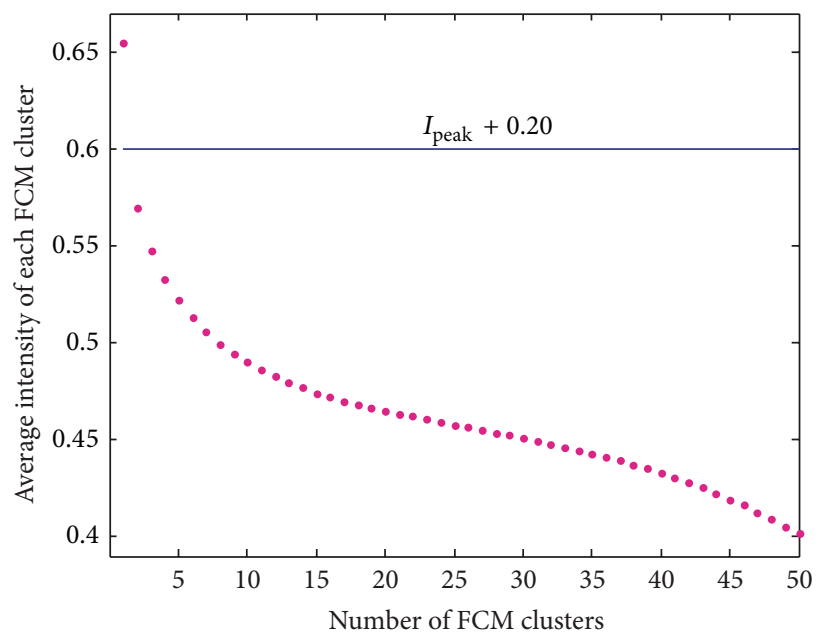

(b)

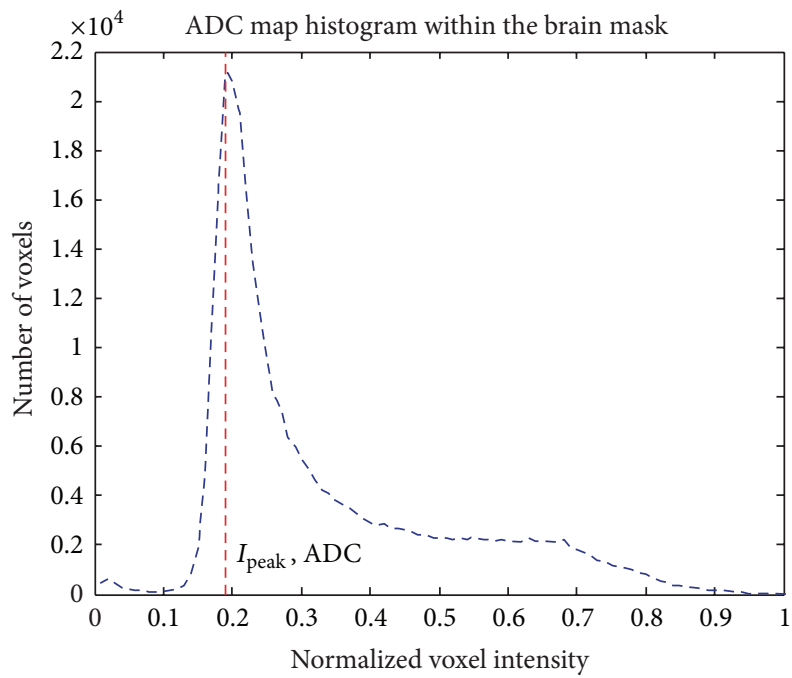

(d)

Figure 3: (a) The histogram of the normalized voxel intensity within the brain mask of a whole-brain DWI. (b) The average normalized intensities of the 50 clusters created in Step 4. (c) The average normalized intensities of the labels from the clusters of candidate voxels of infarct in Step 5. (d) The histogram of the normalized voxel intensity within the brain mask of an ADC map.

to correct for differences due to head movements [18]. The DWI and registered ADC map were normalized so that their intensities were both distributed in a standardized range $(0,1)$. The program we used to run this step was the Statistical Parametric Mapping 8 (SPM8, Wellcome Department of Cognitive Neurology, London, UK).

Step 2 (extracting the brain mask from the whole-brain DWI). The brain mask was extracted from the whole-brain DWI based on the estimation of the inner and outer skull surfaces by using BET (Brain Extraction Tool), a software package developed at FMRIB Centre, University of Oxford, Oxford, United Kingdom [19]. Note that the fractional intensity threshold was set at 0.3 , smaller than the default value 0.5 , to give a larger brain outline estimate, which would completely enclose the brain. On one hand, the brain mask extraction would not eliminate any portion of the cerebral infarcts. On the other hand, no part of the brain skull portion enclosed by the brain outline would be mistaken as an infarct region in the subsequence steps, because there are obvious intensity differences between the cerebral infarcts and brain skull.

Step 3 (preclustering elimination). The histogram of DWI within the brain mask was smoothed by a third-order moving-average filter. The peak of the smoothed histogram was identified. The normalized intensity, denoted by $I_{\text {peak }}$, corresponding to this histographic peak was used as a threshold. The voxels with normalized intensities lower than or equal to $I_{\text {peak }}$ would be eliminated from further processing.

Step 4 (FCM clustering). The remaining voxels after the previous step were divided into 50 clusters by an unsupervised classification with the conventional FCM clustering algorithm. 


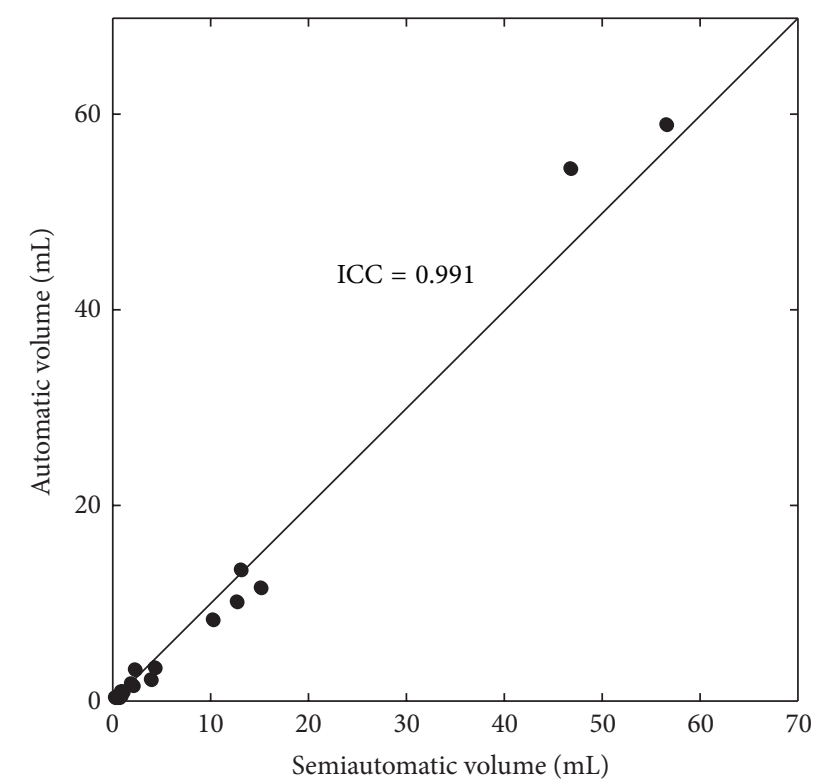

Figure 4: Automatically segmented versus semiautomatically segmented infarct volumes.

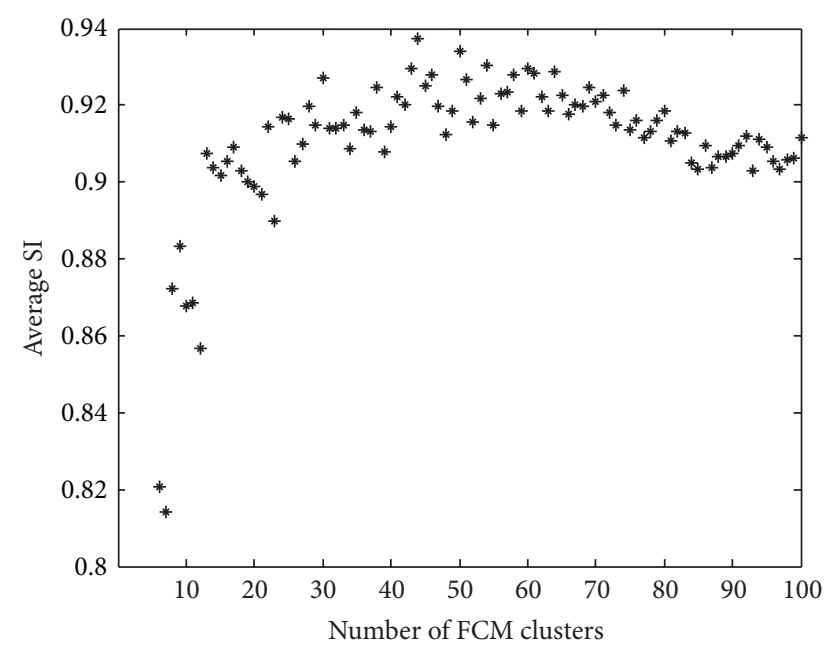

FIGURE 5: The result of the preliminary experiment to determine the FCM cluster number. Each of the average SI values was the average SI of the semiprocedure of the proposed method conducted on the 22 patients.

Step 5 (skimming the clusters for candidate voxels). The clusters with mean normalized intensity larger than the normalized intensity of the histographic peak plus 0.2 , that is, $I_{\text {peak }}+0.2$, were selected. The voxels belonging to these selected clusters would be treated as candidate voxels of infarct in the next step.

Step 6 (eliminating labels with insufficient intensity). Each cluster of candidate voxels of infarct was further divided into one or several labels, at most the same number as the voxel number in that cluster. A label comprised connected voxels. The labels with average normalized intensity lower than or equal to the threshold $\left(I_{\text {peak }}+0.2\right)$ were eliminated from further processing.

Step 7 (eliminating labels with weak edge). The labels with weak edge were eliminated from further processing. The edge map was extracted from normalized DWI by using Canny edge detector [20]. The low and high threshold values were defined as $(0,0.3)$. The parameter of the standard deviation of the Gaussian filter was determined to be 1 .

Step 8 (eliminating candidate labels due to magnetic inhomogeneity). Let $I_{\text {peak,ADC }}$ denote the intensity corresponding to the histographic peak of the ADC map. Further, let $I_{\text {lower mean,ADC }}$ denote the average intensity of the lower-intensity half of all the voxels in a specific label on the ADC map. For this specific label, if the ratio $I_{\text {lower mean,ADC }} / I_{\text {peak,ADC }} \geq 0.5$, this label was considered an artifact caused by magnetic inhomogeneity. All artifacts thus defined were detected and eliminated in this step. Finally, all the voxels in the remaining labels in the remaining clusters were taken to be infarct.

2.3. Performance Evaluation. A voxelwise comparison between the proposed automatic segmentation and semiautomatic segmentation by the experienced neurologist (Chen) [21] gives the four parameters of each patient: true positive (TP), true negative (TN), false positive (FP), and false negative (FN). The sensitivity (Sen.), specificity (Spe.), positive prediction value (PPV), and negative prediction value (NPV) are calculated from the four parameters [22].

The similarity index (SI) is used to indicate the degree of agreement between the infarcts detected by our method and those detected semiautomatically by the neurologist. Its formula is $\mathrm{SI}=2 \times \mathrm{TP} /(2 \times \mathrm{TP}+\mathrm{FP}+\mathrm{FN})$ [23]. In addition, Cohen's kappa coefficient is also calculated. It eliminates the agreement due to random chance and is considered a conservative measure of interrater agreement. Infarct volume was calculated as the summation of the detected infarct area of axial DWIs times the slice thickness [24]. The agreement evaluation of volume measurements of the proposed algorithm was carried out by calculating the intraclass correlation coefficient (ICC) [25].

2.4. Preliminary Experiment. A preliminary experiment was conducted to find the most suitable cluster numbers for the FCM clustering in Step 4. Cluster numbers ranging from 6 to 100 were tested. For each of the tested cluster numbers, a semiprocedure of the proposed method was conducted on each of the 22 recruited patients to obtain an average SI. The semiprocedure consisted of Steps E1 to E7, whereof Steps E1 to E5 were the same as Steps 1 to 5. Steps E6 to E7 were as follows.

Step E6 (selecting infarct labels). Each cluster of candidate voxels of infarct was further divided into one or several labels, at most the same number as the voxel number in that cluster. A label comprised connected voxels. All the labels containing at least one voxel belonging to a semiautomatically demarcated infarct region were selected as infarct labels. 

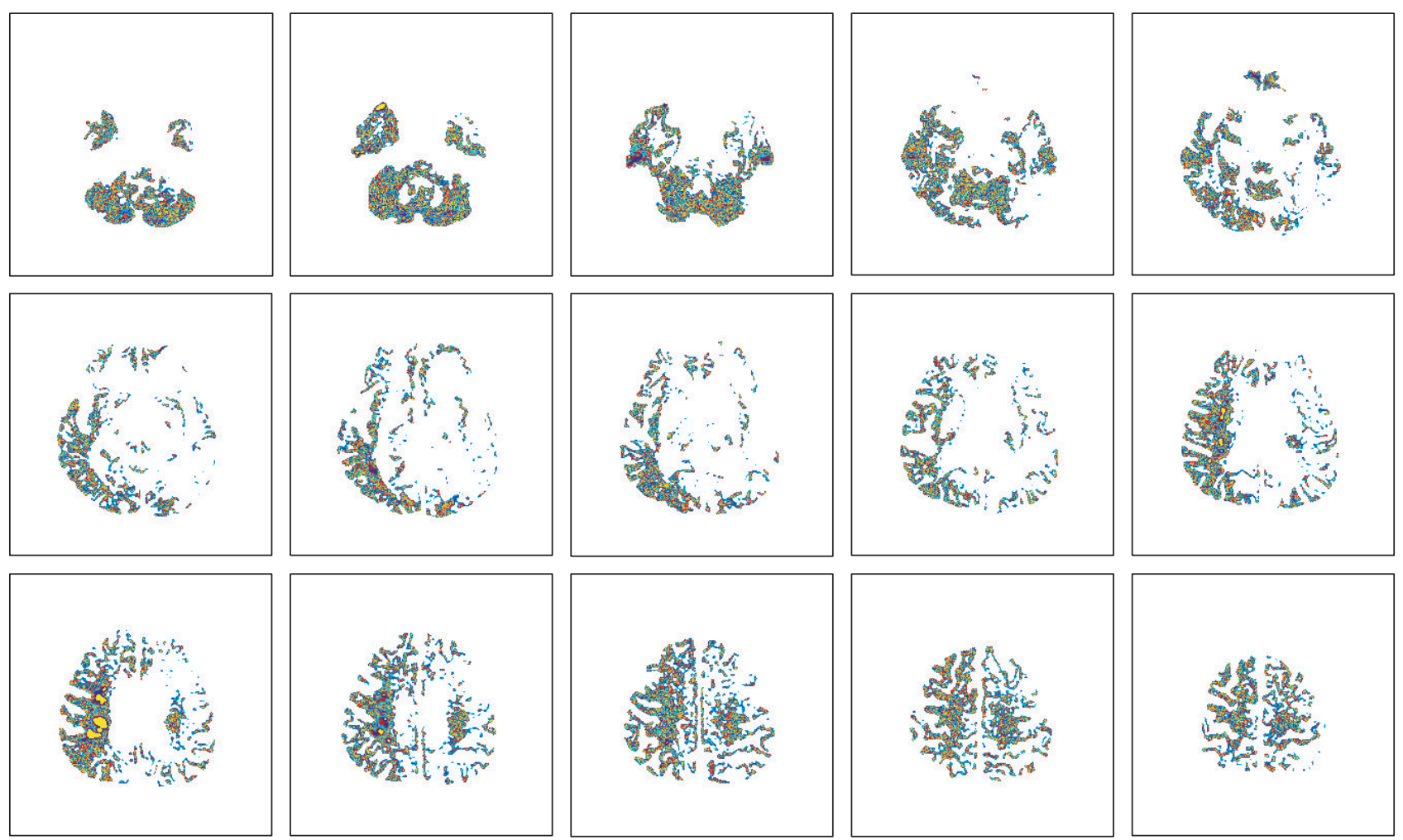

FIGURE 6: Different colors represent the voxels of the 50 different clusters in a whole-brain DWI of patient number 9 in Step 4.

Step E7 (SI calculation). The SI was calculated with the voxelby-voxel comparison between the infarct labels selected in Step 6 and the semiautomatically demarcated infarct regions.

\section{Results}

The exemplary images in Figure 2 illustrate the procedure of the proposed method. Figure 2(a) shows an example of the DWI slice used as the input to the proposed method in Step 1. Figure 2(b) was a slice of the whole-brain mask extracted from the whole-brain DWI in Step 2. Figure 2(c) shows a slice of the output of the preclustering elimination in Step 3. These were the DWI voxels with normalized intensities higher than $I_{\text {peak }}$ within the brain mask. In Figure 2(d), different colors were used to paint the voxels of different clusters in an exemplary DWI slice after the FCM clustering in Step 4. Figure 2(e) shows the corresponding Canny edge detection map. Figure 2(f) shows an example of the final detected infarcts after further processing through Steps 5, 6, 7, and 8. Figure $2(\mathrm{~g})$ is a combination of the detected infarcts and the raw DWI. Figure $2(\mathrm{~h})$ shows the ADC map that was used in Step 8 to eliminate artifact-induced spurious infarcts. Figure 2(i) shows the result of the semiautomatic infarct segmentation by the neurologist on the same input DWI.

Figure 3 shows how the histographic information of the voxel intensity was utilized in the proposed method to facilitate the identification of infarct. The blue curve in Figure 3(a) represents the smoothed histogram of the voxel intensity of a normalized raw DWI. The intensity corresponding to the peak of the histogram is referred to as $I_{\text {peak }}$. In Step 3, all voxels with normalized intensity lower than or equal to $I_{\text {peak }}$ were eliminated because they very unlikely belonged to infarct areas. This preclustering elimination greatly reduced the computation load in the latter steps. Each of the 50 dots in Figure 3(b) represents the average normalized intensity of all the voxels in an individual cluster among the 50 output clusters of the FCM clustering in Step 4. In Step 5, only the clusters with mean normalized intensity higher than or equal to $I_{\text {peak }}+0.2$ were skimmed (selected) as candidate infarct clusters. In the example shown in Figure 3(b), only one cluster became a candidate cluster. Each dot in Figure 3(c) represents the average normalized intensity of an individual label in the candidate cluster(s). The labels with average normalized intensities lower than $I_{\text {peak }}+0.2$ were eliminated in Step 6 . Figure 3(d) shows the histogram of the normalized voxel intensity of the ADC map. It was used in Step 8 to distinguish artifacts due to magnetic inhomogeneity.

Verified with the reference by the experienced neurologist (Chen), our algorithm had high sensitivity, specificity, and SI. Table 1 tabulates the performance indices of our algorithm on each of the 22 subjects. Note that we conducted the proposed method 10 times on each patient's MRI to get a (mean \pm standard deviation), shown in the supplementary table (see Supplementary Material available online at http://dx.doi.org/10.1155/2014/963032), of each performance index. Table 1 only shows the mean values of the performance indices. With total infarct lesion volume ranging from 0.155 to $482.939 \mathrm{~mL}$, the sensitivity was $88.036 \pm 12.117 \%$, 

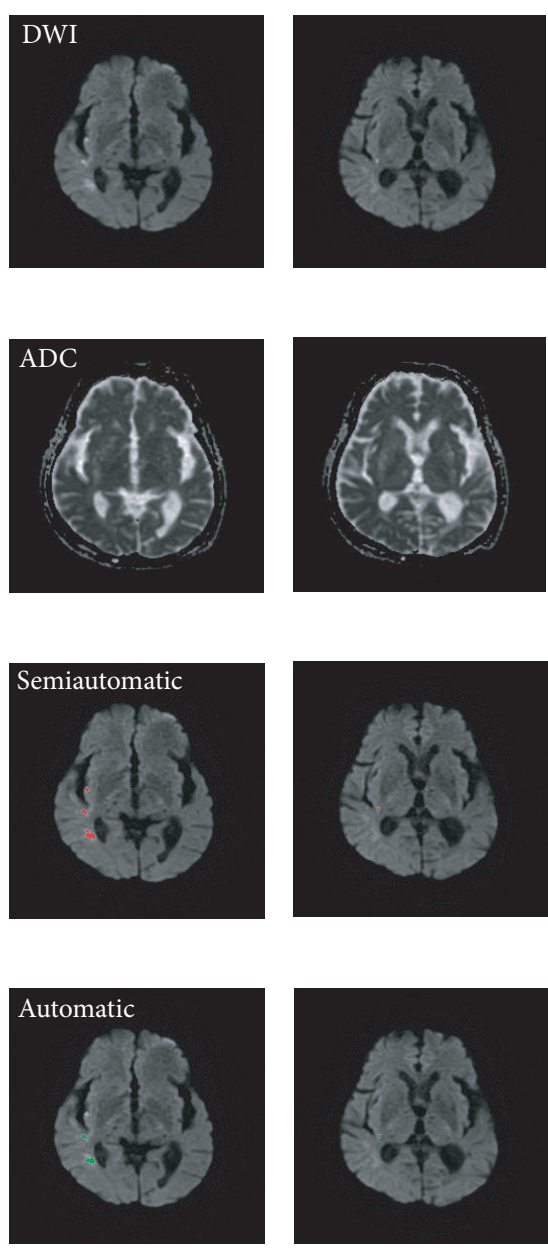
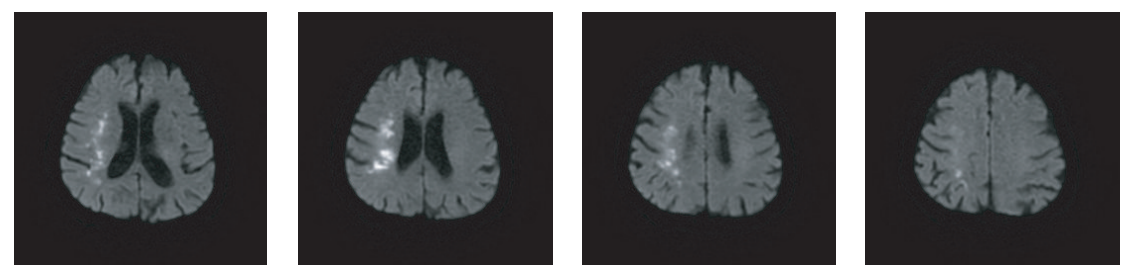

(a)
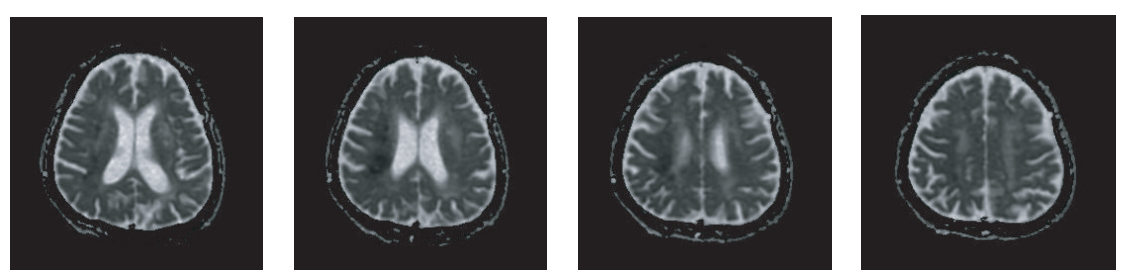

(b)
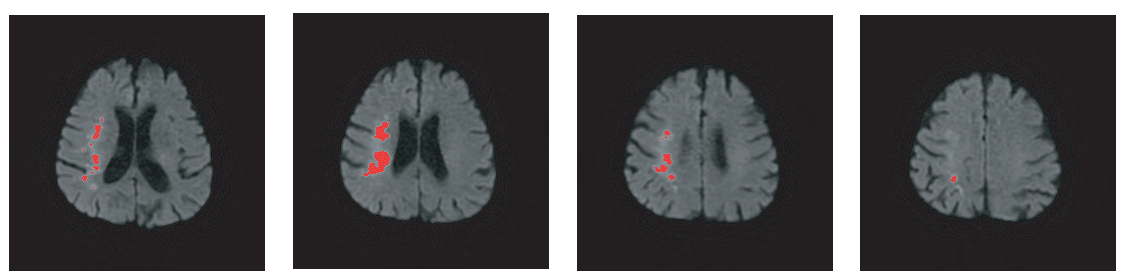

(c)
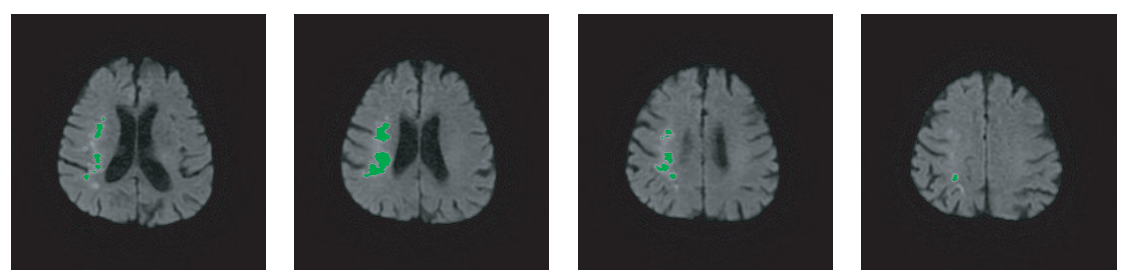

(d)

Figure 7: Illustrating the input and output images of the proposed algorithm using patient number 9 as an example. This figure shows 6 of the 23 slices of the whole-brain MRIs of patient 9. (a) Six axial slices of DWI. (b) Six axial slices of ADC map. (c) Infarct regions, painted red, semiautomatically demarcated by the neurologist. (d) Infarct regions, painted green, detected automatically by the proposed algorithm.

the specificity $99.992 \pm 0.024 \%$, and the SI $89.933 \pm 6.460 \%$. The standard deviations shown in the supplementary table reveal that the variation due to FCM clustering was low and acceptable.

Figure 4 shows the relationship in the infarct volumes obtained by the semiautomatic method and the proposed method. The infarct volumes determined by the proposed method correlated well with those determined by the semiautomatic method with an ICC of 0.991 . Notice that patient number 22 was an outlier, with a much higher infarct volume than those of the other patients. This patient is not included in the plot of Figure 4. The ICC with patient number 22 included was 0.993 .

The result of the preliminary experiment with various output cluster numbers of the FCM clustering algorithm is shown in Figure 5. It demonstrates that the number of the output clusters has substantial influence on the average SI value. Figure 6 illustrates the result of the FCM clustering by using different colors to represent the 50 different clusters in a whole-brain DWI.
To illustrate the effect of the proposed algorithm, the semiautomatic and the proposed automatic segmentation results on patient $\# 9$ are displayed in Figure 7.

It took our personal computer less than 90 seconds to execute from Step 1 through Step 8. However, because the SPM program in Step 1 and the BET program in Step 2 required user intervention, the whole procedure actually took nearly 5 minutes. In the future, if the functions currently executed by the SPM and BET programs are integrated into the main MATLAB program, the whole procedure will finish within 90 seconds.

\section{Discussion}

We reported a high SI of our algorithm. The method proposed by Bhanu Prakash et al. attained 60\% in SI [12]. The SI of their proposed method two years later [10] was improved to $67 \%$. Another method [14] proposed by the same team attained $67.6 \%$ in SI. The method [15] by Shen et al. could attain $87.9 \%$ in the average SI, obtained with simulated lesions. 


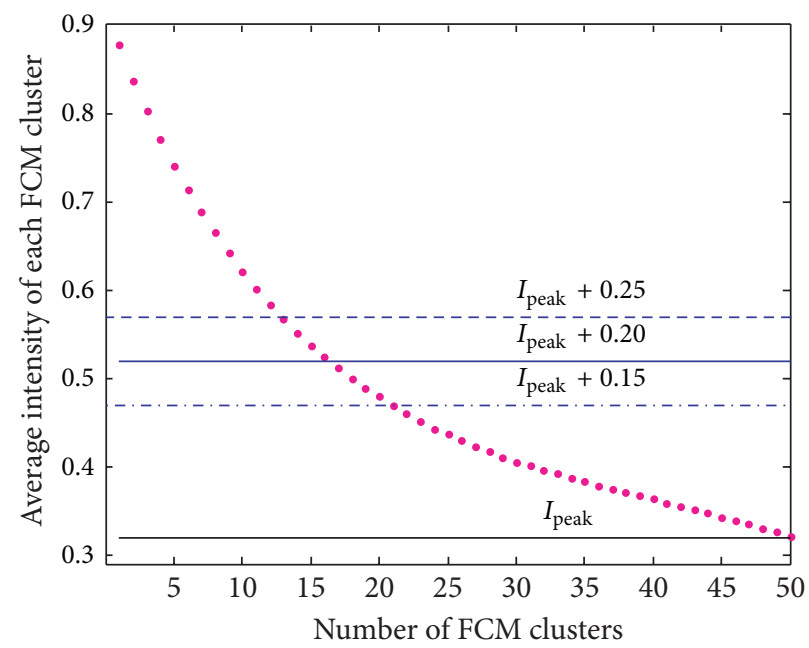

(a)

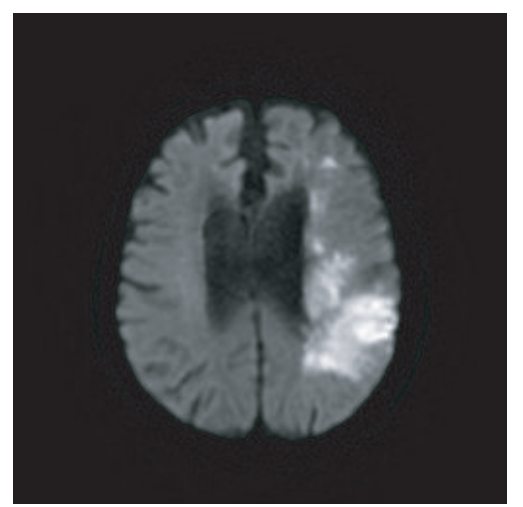

(b)

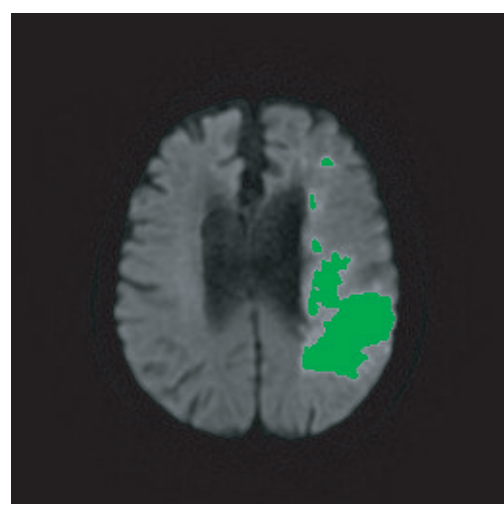

(d)

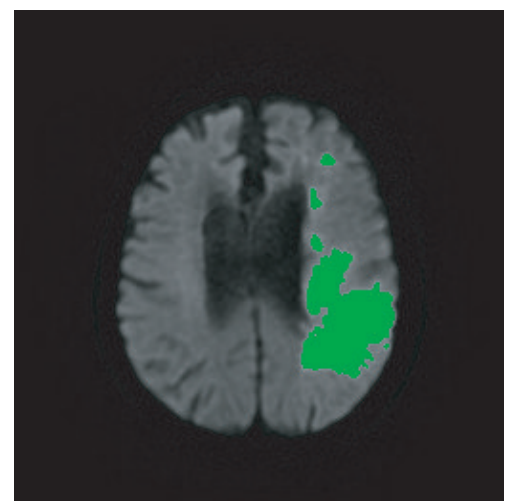

(c)

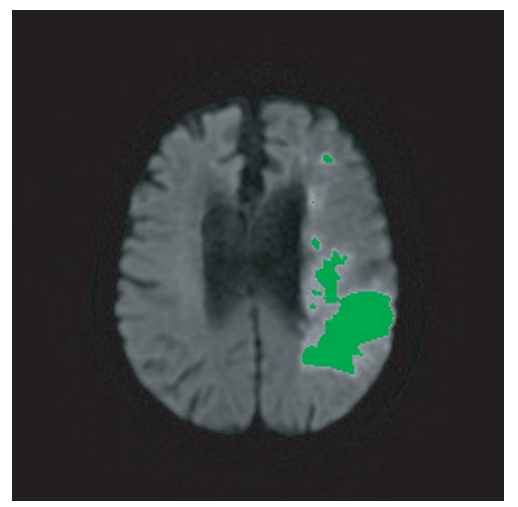

(e)

FIGURE 8: The effect of threshold variation of clusters skimming in Step 5. (a) Each red dot represents the average normalized intensity of an individual cluster among the 50 output clusters of the FCM clustering. (b) The raw DWI. (c) The detected infarct lesions with skimming from $I_{\text {peak }}+0.15$. (d) The detected infarct lesions with skimming from $I_{\text {peak }}+0.2$. (e) The detected infarct lesions with skimming from $I_{\text {peak }}+0.25$. The SI values of the three cases were $88.4 \%, 93.3 \%$, and $99.2 \%$, whereas the sensitivities were $100.0 \%, 99.9 \%$, and $98.4 \%$, respectively.

The method [11] proposed by Li et al. attained SI above $92 \%$. In comparison, the performance of our method in terms of SI is higher than those of Prakash and Gupta's team, similar to the method of Shen et al., and only next to that of the method of $\mathrm{Li}$ et al. It is worth noting that the SI values reported by different research teams were based on different gold standards and calculated from different experiment setups.
Hence, there is no fair comparison among the performances of these methods.

We attribute the high SI of our method to the following key points.

First, we used a self-adaptive threshold, that is, $I_{\text {peak }}$, in the preclustering elimination step (Step 3). The purpose of the preclustering elimination was to increase the efficiency of 


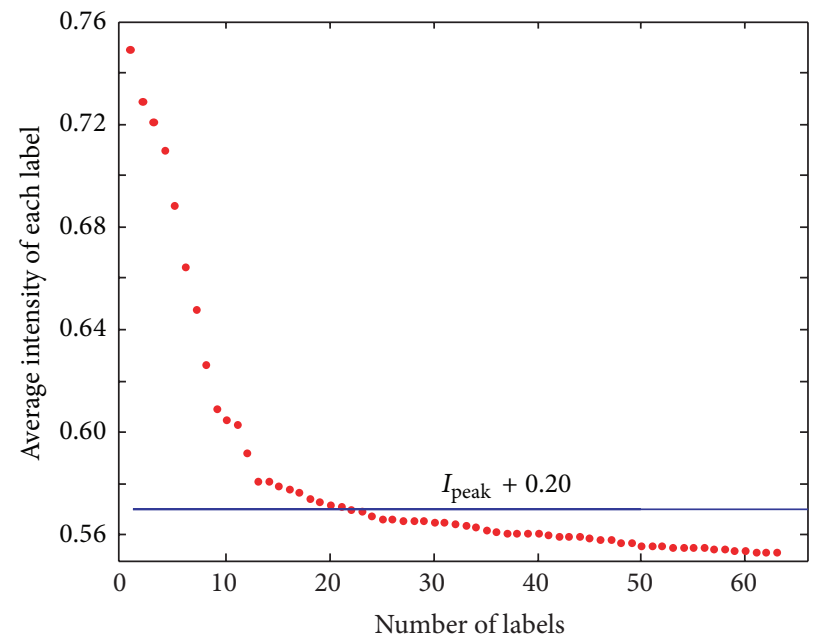

(a)

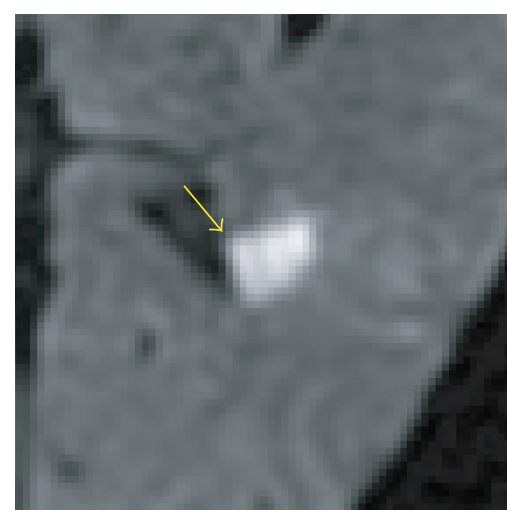

(b)

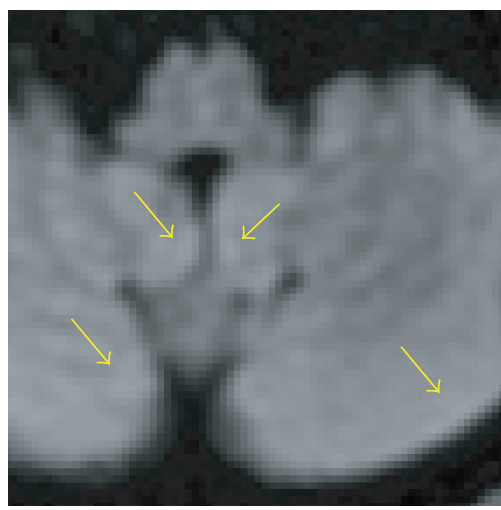

(d)

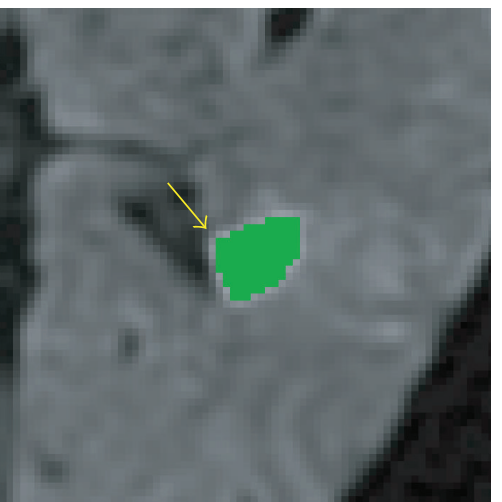

(c)

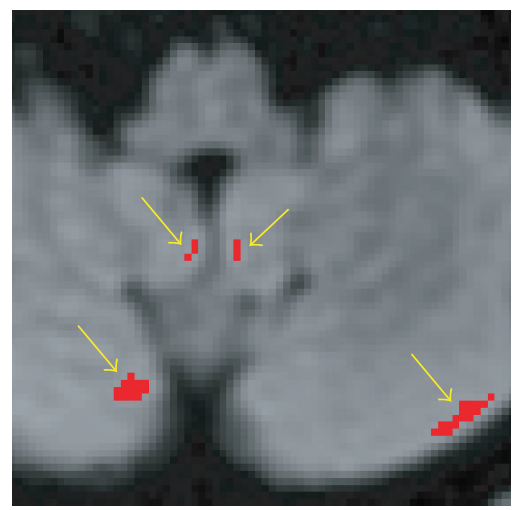

(e)

FIGURE 9: In Step 6, labels in the skimmed cluster(s) would be eliminated if their individual average normalized intensities were lower than or equal to the threshold level, namely, $I_{\text {peak }}+0.2$. (a) The red dots represent the average normalized intensities of the labels in the cluster(s) that had been skimmed in Step 5. The blue line indicates the threshold level in this step. (b) A typical label, pointed to by the arrow, with its average normalized intensity higher than the threshold level would appear white possibly with a faint suburb. (c) Such a label, painted green, was classified as an infarct region. (d) The arrows point to labels with individual average normalized intensities lower than the threshold level. They appeared faint all over. (e) Such labels, painted red, were classified as noninfarct regions. 


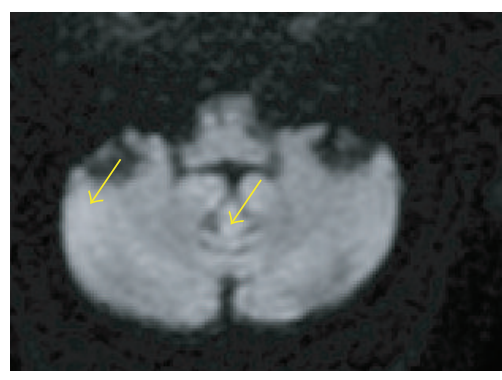

(a)

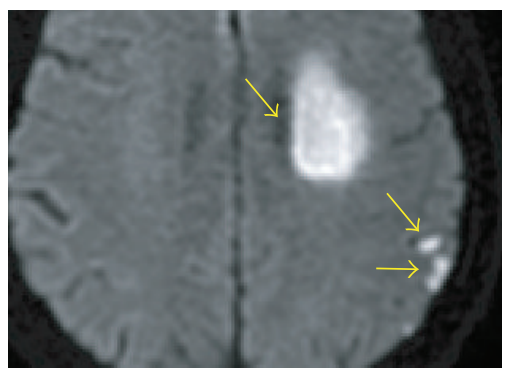

(d)

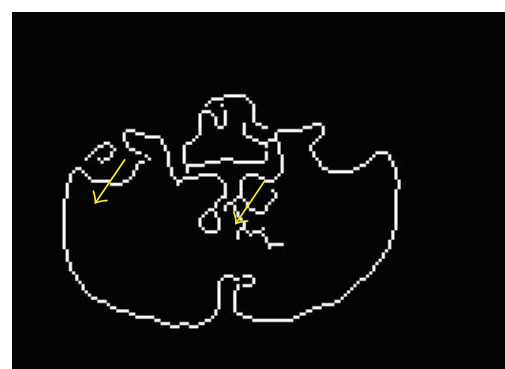

(b)

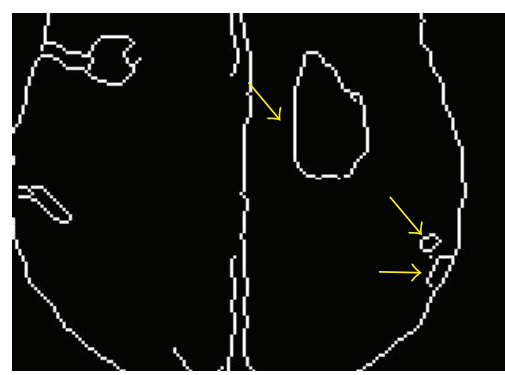

(e)

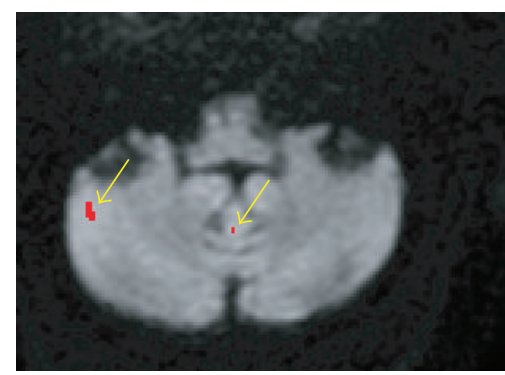

(c)

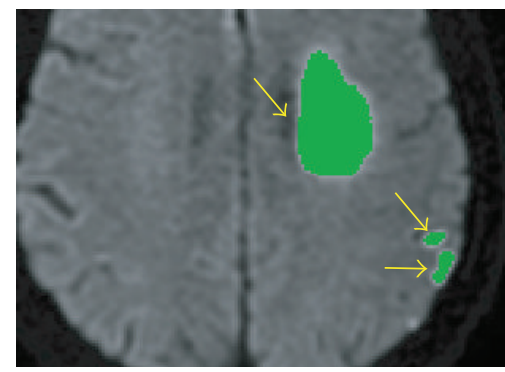

(f)

Figure 10: In Step 7, labels selected in Step 6 would be eliminated if their edges were weak. (a)-(b) The labels pointed to by the arrows were all labels that had been selected in Step 6 for further processing. (c) In the Canny edge detection map, the arrow-pointed-to labels in (a) did not have corresponding edges because their edges were weak. (d) In the Canny edge detection map, the arrow-pointed-to labels in (b) had corresponding edges because their edges were not weak. (e) The arrow-pointed-to labels in (b) were classified as noninfarct regions, painted red. (f) The arrow-pointed-to labels in (b) were classified as infarct regions, painted green.

fuzzy clustering and reduce the computation time. The histographic peak corresponded to the most abundant intensity of the DWI image. The voxels with intensity lower than $I_{\text {peak }}$ were very unlikely to belong to an infarct, so it is safe to eliminate them from further processing. The threshold was self-adaptable in that the $I_{\text {peak }}$ of every patient served the purpose well.

Second, we used an optimal value for the output cluster number of the FCM clustering algorithm in Step 4. It was chosen to be 50 since this was a value that would lead to high SI values, as was demonstrated in Figure 5.

Third, in Step 5, we selected the mean demarcation threshold, $I_{\text {peak }}+0.2$, used by the neurologist as the threshold for skimming the clusters for candidate voxels. As illustrated in Figures 8(c), 8(d), and 8(e), different extents of skimming led to different SI values. We found that the difference between $I_{\text {peak }}$ and the minimum normalized intensity of the infarct regions demarcated semiautomatically by the neurologist averaged around 0.2. In the proposed algorithm, the normalized intensity value that was 0.2 higher than $I_{\text {peak }}$ was selected for the value from which the skimming started. Note that the demarcation threshold $I_{\text {peak }}+0.2$ had been determined based on the statistics of the experienced neurologist's semiautomatic demarcation results. The level of this threshold can be changed to accommodate for different neurologists, scanners, and acquisition parameters. A suitable new threshold can be obtained by statistical analysis on a training set, making the proposed algorithm adoptable to all situations.
Fourth, we eliminated false-positive labels in the candidate clusters. The results before adopting Step 6 had shown that there would be some false-positive infarct regions in the result if all the voxels of the skimmed clusters were taken as infarct. In Step 6, by dividing each skimmed cluster into labels and eliminating labels with low intensity, the false positives could mostly become true negatives. As illustrated in Figure 9(a), the labels with average normalized intensity higher than or equal to $I_{\text {peak }}+0.2$ were retained in the candidates and the others were eliminated. Figure 9(b) exemplifies a true-positive infarct label, which looked white and might have a faint suburb. Such a label was classified as an infarct region, painted green in Figure 9(c). Figure 9(d) shows some labels with average normalized intensity lower than $I_{\text {peak }}+0.2$; these labels looked faint all over. Such labels were classified as noninfarct regions, painted red in Figure 9(e).

Fifth, edge detection was used to further eliminate labels with weaker edges. The results of Step 6 still contained a few false-positive labels. That is to say, some noninfarct labels had sufficient normalized intensity to pass the decisions in Steps 5 and 6. However, these false-positive labels had weaker edges than real infarct labels had. Step 7 used this edge for eliminating the false-positive labels. Figure 10 demonstrates the function of this step with real examples.

Sixth, we detected and eliminated the false-positive labels due to magnetic-inhomogeneity artifacts. The result of Step 7 still contained false-positive labels, which were actually artifacts caused by the magnetic susceptibility differences between adjacent air and cerebrospinal fluid structures and 


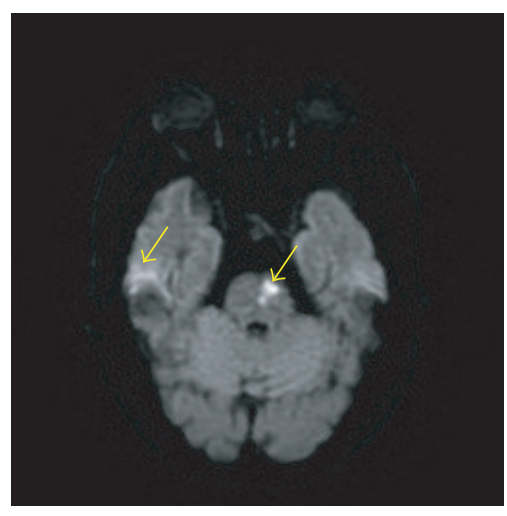

(a)

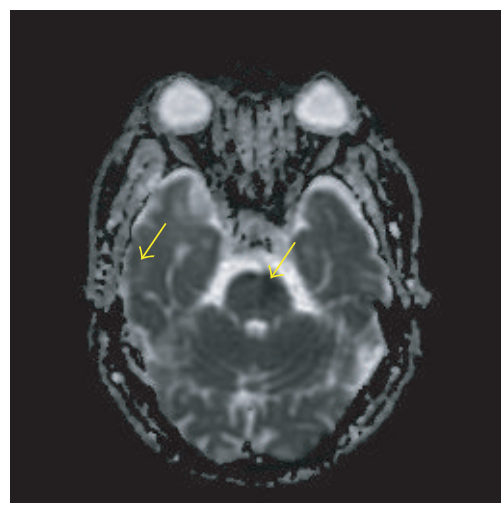

(b)

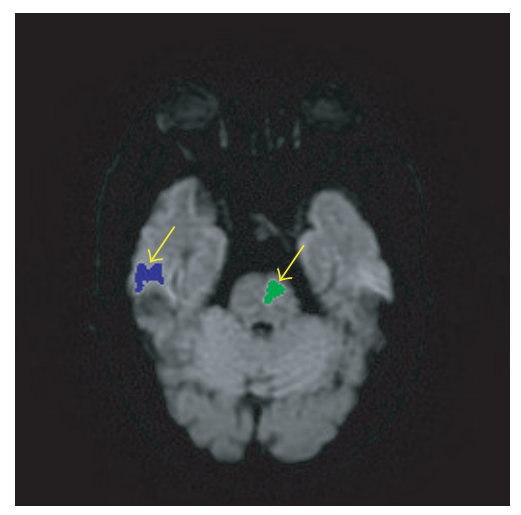

(c)

FIGURE 11: In Step 8, candidate labels due to magnetic inhomogeneity were eliminated. (a) In this example, the two labels pointed by arrows had intensities higher than $I_{\text {peak }}+0.2$ and appeared equally bright in the DWI. (b) The two labels had different intensities in the ADC map. (c) The artifact (blue) was differentiated from the infarct (green) in Step 8.

the surrounding soft tissues with echo-planar imaging techniques [26]. In the DWI, the magnetic inhomogeneity created artifacts with intensities commensurate with those of infarcts. It had been a difficult task to eliminate the artifacts [12]. However, in the ADC map, the artifact intensity was higher than the infarct intensity. This property was used in Step 8 to detect and eliminate the artifacts caused by magnetic inhomogeneity. Figure 11 illustrates this phenomenon.

The proposed method had the second lowest SI (79.722\%), despite of $100.000 \%$ sensitivity, for patient number 13 among the 22 patients. That was actually due to inconsistent selection of threshold by the neurologist. Notice from Table 1 that the demarcation boundary selected by the neurologist for patient number 13 was $I_{\text {peak }}+0.31$, which was much higher than the average value. In other words, the neurologist included fewer voxels into infarct than usual. The demarcation boundary selected by the neurologist for patient number 14 was $I_{\text {peak }}+0.14$, much lower than the average value. That is to say, more voxels than usual were included into the infarct by the neurologist. That was why the proposed method had the lowest SI (74.359\%) and the lowest sensitivity (59.444\%) for patient number 14 among all the patients. These cases demonstrated the inconsistency in the semiautomatic way of segmentation. In contrast, the proposed method attained consistent segmentation, because it used the same value as the threshold in all cases.

Although the performance of the proposed method has been satisfactory, an even higher SI is still desirable. At present, the magnetic inhomogeneity does pose a limitation in further increasing the accuracy of infarct detection. To attain higher SI, it will be necessary to find a smarter way than the method we use in Step 8 to identify artifacts due to magnetic inhomogeneity.

This proposed infarct detection method will also be useful for the development of the automatic detection of white matter lesions. It can increase the accuracy of white matter lesion detection by excluding infarct lesions, which could be mistook for white matter lesions easily.

\section{Conclusion}

This proposed algorithm for acute infarct segmentation provides a prompt calculation of acute infarct volume from the DWI and ADC map. The infarct detection was achieved with fuzzy clustering that divided the DWI into ordered clusters based on voxel intensities. Under careful scrutiny on the intensity spectrum, candidate clusters were skimmed and deceptive labels further eliminated. Additionally, the ADC 
TABLE 1: Demographics and the statistics of the computer-assisted cerebral infarct segmentation results of individual patients after repeating the proposed method for 10 times.

\begin{tabular}{|c|c|c|c|c|c|c|c|c|c|c|c|c|}
\hline \multirow{2}{*}{ Patient } & \multirow{2}{*}{ Sex } & \multirow{2}{*}{ Age } & \multirow{2}{*}{ Demar. } & \multicolumn{2}{|c|}{ Total infarct volume (mL) } & \multirow{2}{*}{$\Delta$ Vol. } & \multirow{2}{*}{ SI (\%) } & \multirow{2}{*}{ Kappa (\%) } & \multirow{2}{*}{ Sen. $(\%)$} & \multirow{2}{*}{ Spe. (\%) } & \multirow{2}{*}{ PPV (\%) } & \multirow{2}{*}{ NPV (\%) } \\
\hline & & & & Semi-auto. & Auto. & & & & & & & \\
\hline 1 & $\mathrm{M}$ & 73 & 0.27 & 0.155 & 0.212 & $36.6 \%$ & 84.661 & 84.661 & 100.000 & 99.999 & 73.529 & 100.000 \\
\hline 2 & $\mathrm{M}$ & 80 & 0.19 & 0.358 & 0.377 & $5.3 \%$ & 97.438 & 97.438 & 100.000 & 100.000 & 95.014 & 100.000 \\
\hline 3 & $\mathrm{~F}$ & 69 & 0.21 & 0.399 & 0.381 & $-4.4 \%$ & 97.716 & 97.716 & 95.556 & 100.000 & 100.000 & 100.000 \\
\hline 4 & $\mathrm{~F}$ & 84 & 0.22 & 0.474 & 0.378 & $-20.3 \%$ & 86.835 & 86.834 & 78.037 & 100.000 & 98.349 & 99.998 \\
\hline 5 & $\mathrm{~F}$ & 70 & 0.2 & 0.501 & 0.337 & $-32.7 \%$ & 91.662 & 91.662 & 93.398 & 100.000 & 91.723 & 100.000 \\
\hline 6 & $\mathrm{M}$ & 63 & 0.19 & 0.545 & 0.451 & $-17.2 \%$ & 90.413 & 90.412 & 82.764 & 100.000 & 100.000 & 99.999 \\
\hline 7 & $\mathrm{M}$ & 55 & 0.2 & 0.612 & 0.471 & $-22.9 \%$ & 82.684 & 82.683 & 73.190 & 100.000 & 95.072 & 99.998 \\
\hline 8 & F & 66 & 0.15 & 0.644 & 0.479 & $-25.6 \%$ & 85.332 & 85.331 & 74.436 & 100.000 & 100.000 & 99.998 \\
\hline 9 & $\mathrm{M}$ & 61 & 0.21 & 0.796 & 0.895 & $12.5 \%$ & 94.143 & 94.142 & 100.000 & 99.999 & 88.938 & 100.000 \\
\hline 10 & $\mathrm{M}$ & 64 & 0.19 & 1.003 & 1.019 & $1.6 \%$ & 98.927 & 98.927 & 99.710 & 100.000 & 98.171 & 100.000 \\
\hline 11 & $\mathrm{~F}$ & 75 & 0.25 & 1.675 & 1.794 & $7.1 \%$ & 94.373 & 94.372 & 97.619 & 99.998 & 91.568 & 99.999 \\
\hline 12 & $\mathrm{~F}$ & 56 & 0.19 & 1.966 & 1.497 & $-23.9 \%$ & 86.438 & 86.434 & 76.140 & 100.000 & 100.000 & 99.992 \\
\hline 13 & F & 74 & 0.31 & 2.141 & 3.232 & $51.0 \%$ & 79.722 & 79.715 & 100.000 & 99.984 & 66.312 & 100.000 \\
\hline 14 & F & 86 & 0.14 & 3.749 & 2.228 & $-40.6 \%$ & 74.359 & 74.349 & 59.444 & 100.000 & 100.000 & 99.977 \\
\hline 15 & $\mathrm{M}$ & 76 & 0.19 & 4.143 & 3.448 & $-16.8 \%$ & 85.827 & 85.819 & 78.898 & 99.997 & 95.789 & 99.987 \\
\hline 16 & F & 87 & 0.17 & 10.108 & 8.351 & $-17.4 \%$ & 90.453 & 90.440 & 82.621 & 100.000 & 100.000 & 99.975 \\
\hline 17 & $\mathrm{~F}$ & 83 & 0.16 & 12.657 & 10.088 & $-20.3 \%$ & 88.603 & 88.582 & 79.662 & 100.000 & 99.952 & 99.958 \\
\hline 18 & F & 57 & 0.19 & 13.063 & 13.492 & $3.3 \%$ & 97.656 & 97.652 & 99.254 & 99.992 & 96.124 & 99.999 \\
\hline 19 & $\mathrm{M}$ & 72 & 0.19 & 15.014 & 11.611 & $-22.7 \%$ & 87.181 & 87.159 & 77.332 & 100.000 & 99.992 & 99.955 \\
\hline 20 & $\mathrm{M}$ & 80 & 0.24 & 46.828 & 54.497 & $16.4 \%$ & 92.426 & 92.374 & 99.990 & 99.894 & 85.933 & 100.000 \\
\hline 21 & $\mathrm{M}$ & 74 & 0.22 & 56.517 & 59.147 & $4.7 \%$ & 97.526 & 97.503 & 99.793 & 99.956 & 95.365 & 99.998 \\
\hline 22 & $\mathrm{M}$ & 91 & 0.1 & 482.939 & 429.534 & $-11.1 \%$ & 94.147 & 93.675 & 88.942 & 100.000 & 100.000 & 99.056 \\
\hline Mean & & 72.5 & 0.20 & & & $-6.2 \%$ & 89.933 & 89.904 & 88.036 & 99.992 & 94.174 & 99.949 \\
\hline STDEV & & 10.4 & 0.04 & & & $22.3 \%$ & 6.460 & 6.446 & 12.117 & 0.024 & 8.886 & 0.200 \\
\hline
\end{tabular}

Note: Demar.: the lowest intensity, above $I_{\text {peak }}$, of the infarcts demarcated by the neurologist; SI: similarity index; Sen.: sensitivity; Spe.: specificity; PPV: positive predictive value; NPV: negative predictive value; STDEV: standard deviation; $\Delta$ Vol. $=($ Semi-auto. vol. - Auto. vol. $) /$ Semi-auto. vol.

map was used to help identify spurious infarcts that were actually artifacts caused by magnetic inhomogeneity. This method attained high similarity indices. With the semiautomatic segmentation by the experienced neurologist for comparison, this automatic method attained high similarity indices.

Our future research will emphasize finding a more effective way to deal with the magnetic inhomogeneity to attain a higher accuracy in infarct segmentation. Applying the proposed infarct detection method to facilitate accurate computer-assisted segmentation of white matter lesions will also be our future effort.

\section{Conflict of Interests}

The authors declare that there is no conflict of interests regarding the publication of this paper.

\section{Acknowledgments}

The study is supported by a grant (NCU/LSH-101-A-005) from 2012 Annual National Central University and Landseed Hospital Joint R \& D Project, Taiwan and in part by a grant
(NSC 101-2221-E-008-015-MY3) from the National Science Council, Taiwan.

\section{References}

[1] S. C. Smith Jr., "Reducing the global burden of ischemic heart disease and stroke: a challenge for the cardiovascular community and the United Nations," Circulation, vol. 124, no. 3, pp. 278-279, 2011.

[2] G. Vogt, R. Laage, A. Shuaib, and A. Schneider, "Initial lesion volume is an independent predictor of clinical stroke outcome at day 90: an analysis of the Virtual International Stroke Trials Archive (VISTA) database," Stroke, vol. 43, no. 5, pp. 1266-1272, 2012.

[3] S. F. Zaidi, A. Aghaebrahim, X. Urra, M. A. Jumaa, B. Jankowitz, and M. Hammer, "Final infarct volume is a stronger predictor of outcome than recanalization in patients with proximal middle cerebral artery occlusion treated with endovascular therapy," Stroke, vol. 43, no. 12, pp. 3238-3244, 2012.

[4] S. Rangaraju, K. Owada, A. R. Noorian, R. G. Nogueira, F. Nahab, and B. A. Glenn, "Comparison of final infarct volumes in patients who received endovascular therapy or intravenous thrombolysis for acute intracranial large-vessel occlusions," Journal of the American Medical Association, vol. 70, no. 7, pp. 831-836, 2013. 
[5] M. Al-Khaled, C. Matthis, T. F. Munte, and J. Eggers, "QugSS2Study. The incidence and clinical predictors of acute infarction in patients with transient ischemic attack using MRI including DWI," Neuroradiology, vol. 55, no. 2, pp. 157-163, 2013.

[6] M. Lettau and M. Laible, "3-T high-b-value diffusion-weighted MR imaging in hyperacute ischemic stroke," Journal of Neuroradiology, vol. 40, no. 3, pp. 149-157, 2013.

[7] H. L. Lutsep, G. W. Albers, A. DeCrespigny, G. N. Kamat, M. P. Marks, and M. E. Moseley, "Clinical utility of diffusionweighted magnetic resonance imaging in the assessment of ischemic stroke," Annals of Neurology, vol. 41, no. 5, pp. 574580, 1997.

[8] V. F. Newcombe, T. Das, and J. J. Cross, "Diffusion imaging in neurological disease," Journal of Neurology, vol. 260, no. 1, pp. 335-342, 2013.

[9] N. Perez de la Ossa, M. Hernandez-Perez, S. Domenech, P. Cuadras, A. Massuet, and M. Millan, "Hyperintensity of distal vessels on FLAIR is associated with slow progression of the infarction in acute ischemic stroke," Cerebrovascular Diseases, vol. 34, no. 5-6, pp. 376-384, 2012.

[10] K. N. Bhanu Prakash, V. Gupta, H. Jianbo, and W. L. Nowinski, "Automatic processing of diffusion-weighted ischemic stroke images based on divergence measures: slice and hemisphere identification, and stroke region segmentation," International Journal of Computer Assisted Radiology and Surgery, vol. 3, no. 6, pp. 559-570, 2008.

[11] W. Li, J. Tian, E. Li, and J. Dai, "Robust unsupervised segmentation of infarct lesion from diffusion tensor MR images using multiscale statistical classification and partial volume voxel reclassification," NeuroImage, vol. 23, no. 4, pp. 1507-1518, 2004.

[12] K. Bhanu Prakash, V. Gupta, M. Bilello, N. J. Beauchamp, and W. L. Nowinski, "Identification, segmentation, and image property study of acute infarcts in diffusion-weighted images by using a probabilistic neural network and adaptive gaussian mixture model," Academic Radiology, vol. 13, no. 12, pp. 1474-1484, 2006.

[13] N. Hevia-Montiel, J. R. Jimenez-Alaniz, V. Medina-Banuelos et al., "Robust nonparametric segmentation of infarct lesion from diffusion-weighted MR images," in Proceedings of the IEEE Engineering in Medicine and Biology Society, pp. 2102-2105, 2007.

[14] V. Gupta, B. Prakash, and W. L. Nowinski, "Automatic and rapid identification of infarct slices and hemisphere in DWI scans," Academic Radiology, vol. 15, no. 1, pp. 24-39, 2008.

[15] S. Shen, A. J. Szameitat, and A. Sterr, "Detection of infarct lesions from single MRI modality using inconsistency between voxel intensity and spatial location-a 3-D automatic approach," IEEE Transactions on Information Technology in Biomedicine, vol. 12, no. 4, pp. 532-540, 2008.

[16] J. C. Bezdek, Pattern Recognition with Fuzzy Objective Function Algorithms, Kluwer Academic Publishers, Norwell, Mass, USA, 1981.

[17] F.-I. Hsieh, L.-M. Lien, S.-T. Chen et al., "Get with the guidelines-stroke performance indicators: surveillance of stroke care in the taiwan stroke registry: get with the guidelines-stroke in Taiwan," Circulation, vol. 122, no. 11, pp. 1116-1123, 2010.

[18] F. Maes, A. Collignon, D. Vandermeulen, G. Marchal, and P. Suetens, "Multimodality image registration by maximization of mutual information," IEEE Transactions on Medical Imaging, vol. 16, no. 2, pp. 187-198, 1997.

[19] S. M. Smith, "Fast robust automated brain extraction," Human Brain Mapping, vol. 17, no. 3, pp. 143-155, 2002.
[20] J. Canny, "A computational approach to edge detection," IEEE Transactions on Pattern Analysis and Machine Intelligence, vol. 8, no. 6, pp. 679-698, 1986.

[21] Y. W. Chen, M. E. Gurol, J. Rosand et al., "Progression of white matter lesions and hemorrhages in cerebral amyloid angiopathy," Neurology, vol. 67, no. 1, pp. 83-87, 2006.

[22] H.-H. Chang, A. H. Zhuang, D. J. Valentino, and W.-C. $\mathrm{Chu}$, "Performance measure characterization for evaluating neuroimage segmentation algorithms," NeuroImage, vol. 47, no. 1, pp. 122-135, 2009.

[23] L. R. Dice, "Measures of the amount of ecologic association between species," Ecology, vol. 26, no. 3, pp. 297-302, 1945.

[24] R. G. Steen, T. Emudianughe, M. Hunte et al., "Brain volume in pediatric patients with sickle cell disease: evidence of volumetric growth delay?" American Journal of Neuroradiology, vol. 26, no. 3, pp. 455-462, 2005.

[25] K. O. McGraw and S. P. Wong, "Forming inferences about some intraclass correlation coefficients," Psychological Methods, vol. 1, no. 1, pp. 30-46, 1996.

[26] P. Marcoň, K. Bartušek, R. Koř́ínek, and Z. Dokoupil, “Correction of artifacts in diffusion-weighted MR images," in Proceedings of the 34th International Conference on Telecommunications and Signal Processing (TSP '11), pp. 391-397, August 2011. 


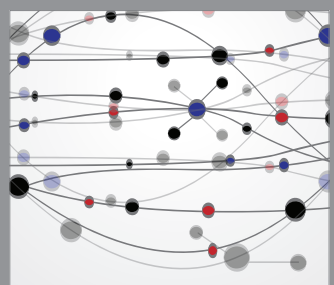

The Scientific World Journal
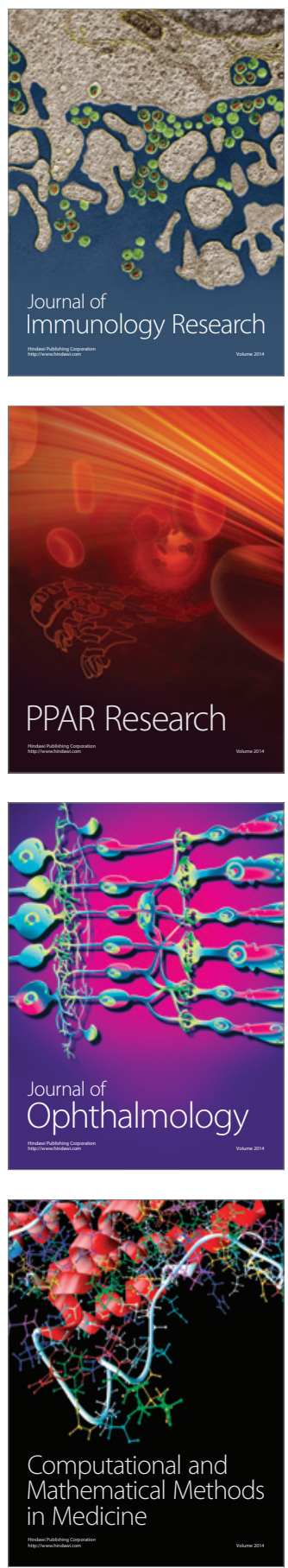

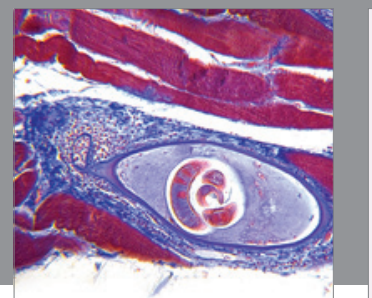

Gastroenterology

Research and Practice
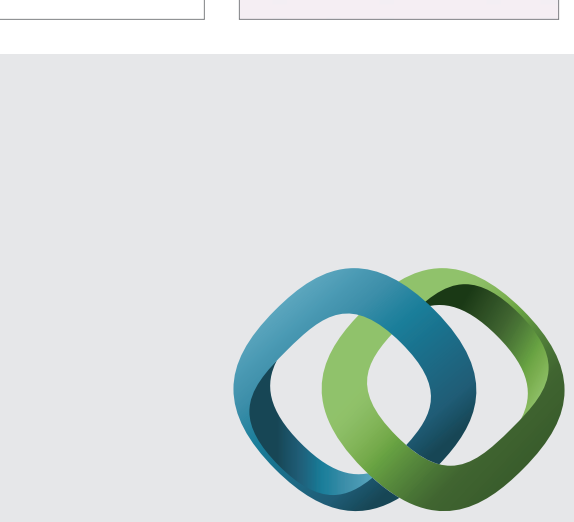

\section{Hindawi}

Submit your manuscripts at

http://www.hindawi.com
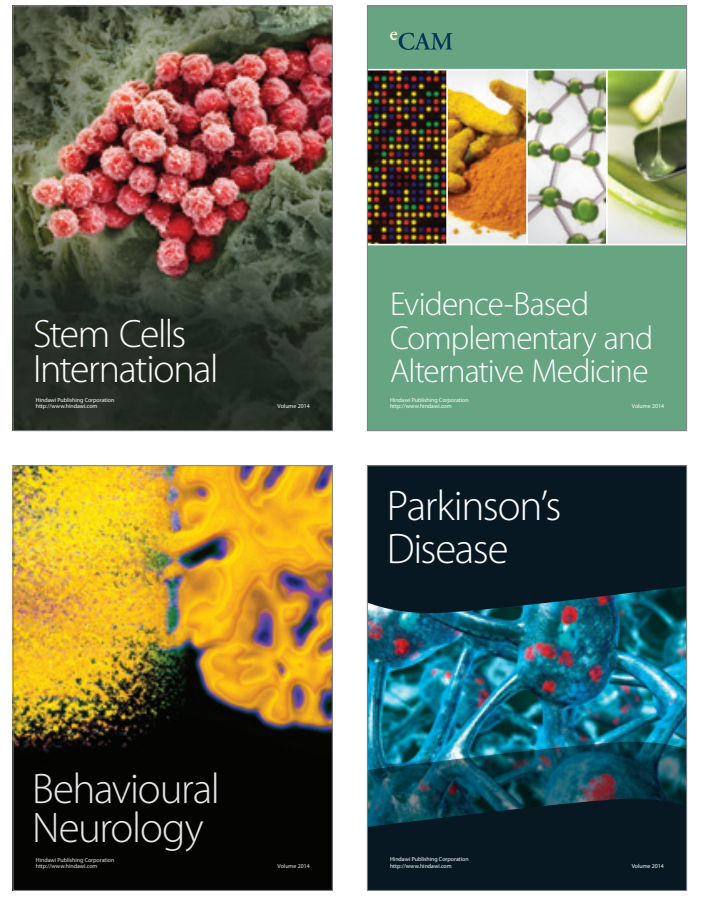
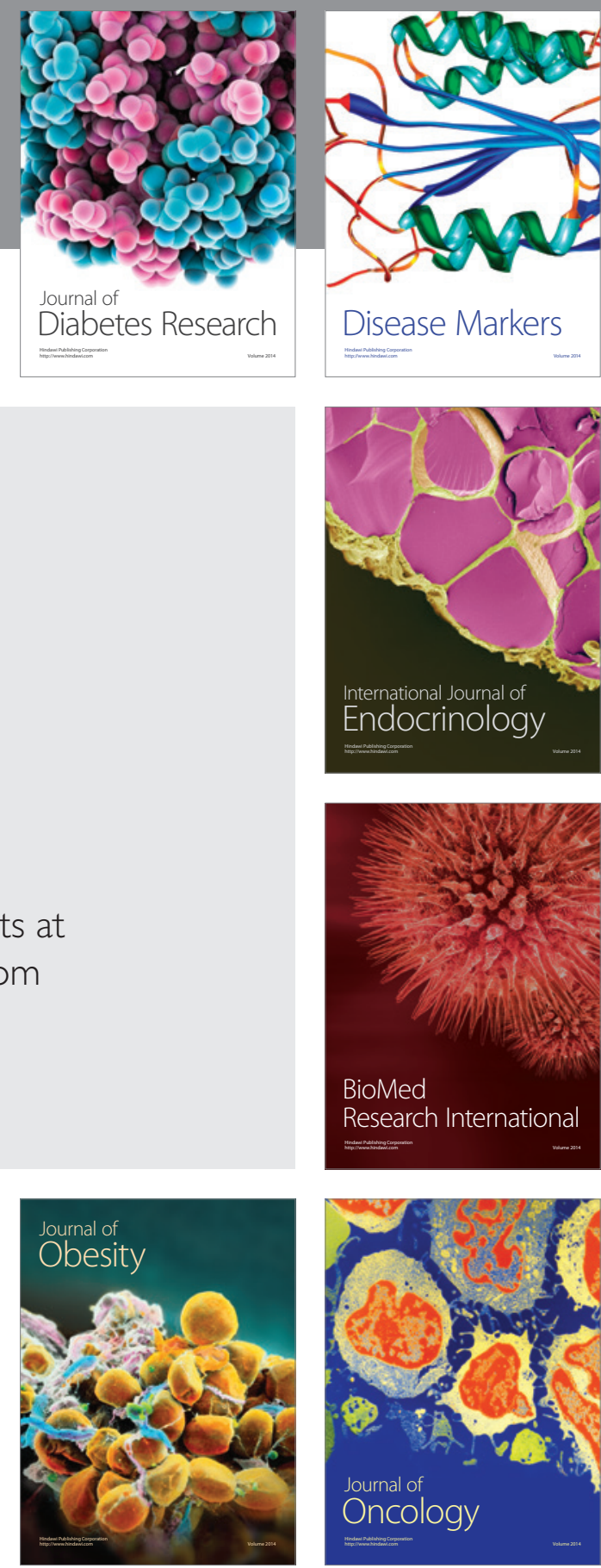

Disease Markers
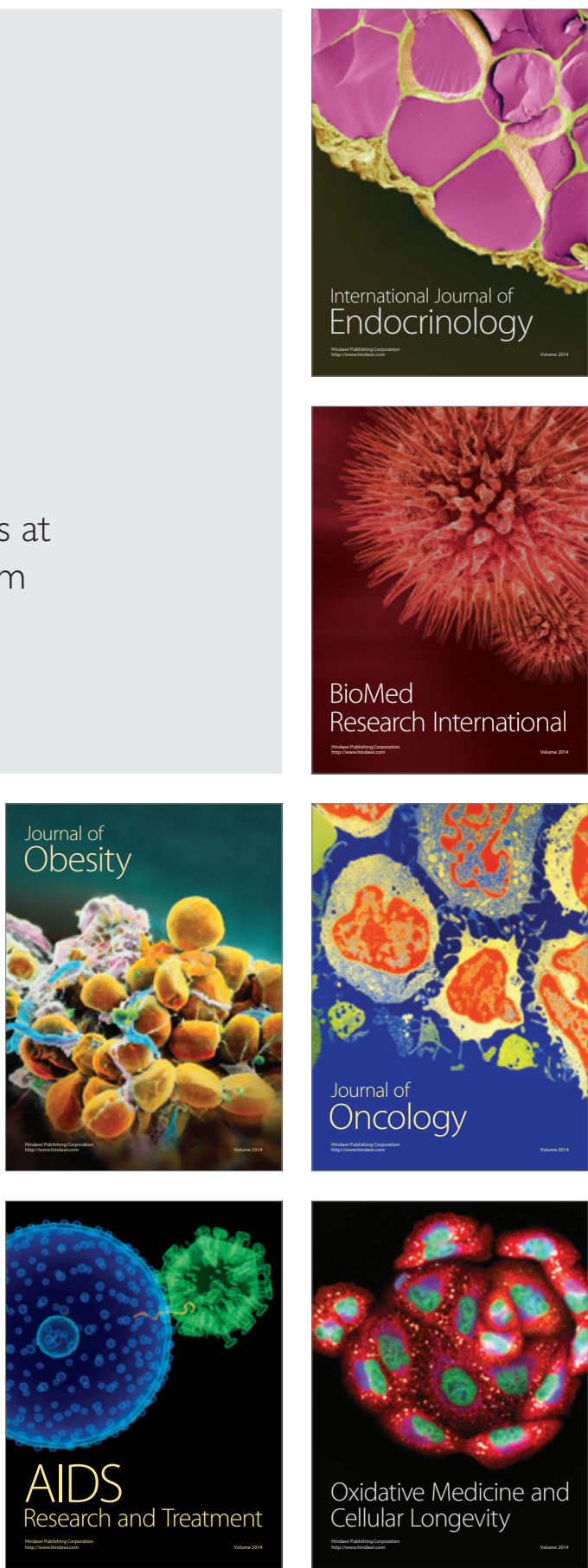